\title{
Global analysis of neutrino oscillation experiments
}

\section{Christoph Andreas Ternes*†}

Institut de Física Corpuscular (CSIC-Universitat de València), Parc Cientific de la UV

C/ Catedratico José Beltrán, 2, E-46980 Paterna (València), Spain

E-mail: chterneseific.uv.es

We present the results of our global fit to neutrino oscillation data. We show the obtained values of the oscillation parameters and discuss the remaining open issues in neutrino oscillations, such as $\mathrm{CP}$ violation, atmospheric octant problem and neutrino mass ordering. To determine the neutrino mass ordering we combine the oscillation results also with data from decay experiments and cosmological observations.

The 20th International Workshop on Neutrinos (NuFact2018)

12-18 August 2018

Blacksburg, Virginia

\footnotetext{
* Speaker.

${ }^{\dagger}$ I would like to thank Mariam Tórtola for helping with these proceedings. Work supported by the FPI fellowship BES-2015-073593 and L'Oréal-UNESCO-Foundation.
} 


\section{Neutrino oscillations}

Since the discovery of neutrino oscillations approximately 20 years ago, experiments have become more and more powerful, bringing the oscillation studies into the precision era. The transition probability from a flavor $\alpha$ to a flavor $\beta$ is described by $P_{\alpha \beta}(E, L)=\sum_{k, j} U_{\alpha k}^{*} U_{\beta k} U_{\alpha j} U_{\beta j}^{*} e^{i \frac{\Delta m_{k j}^{2}}{2 E} L}$, where $E$ is the energy of the neutrino and $L$ the distance traveled by it. The mixing matrix $U$ can be parameterized as

$$
U=\left(\begin{array}{ccc}
1 & 0 & 0 \\
0 & c_{23} & s_{23} \\
0 & -s_{23} & c_{23}
\end{array}\right)\left(\begin{array}{ccc}
c_{13} & 0 & s_{13} e^{-i \delta} \\
0 & 1 & 0 \\
-s_{13} e^{i \delta} & 0 & c_{13}
\end{array}\right)\left(\begin{array}{ccc}
c_{12} & s_{12} & 0 \\
-s_{12} & c_{12} & 0 \\
0 & 0 & 1
\end{array}\right),
$$

where $s_{i j}=\sin \theta_{i j}$ and $c_{i j}=\cos \theta_{i j}$. Therefore, we need six parameters to describe neutrino oscillations: the two mass splittings $\Delta m_{31}^{2}, \Delta m_{21}^{2}$, the three mixing angles $\theta_{12}, \theta_{13}, \theta_{23}$ and the CP-phase $\delta$. While many experiments measure some of the parameters rather well, a combined global fit can provide better determinations, since the advances and sensitivities of all experiments can be used in this way. In Ref. [1] we have performed this combination to extract the oscillation parameters from all available data so far. The combined global analysis leads to the values summarized in Tab. 1 and plotted in Fig. 1, where we marginalized over all the other five parameters. In the following we will shortly address the remaining unknowns in neutrino oscillations.

\begin{tabular}{lccc}
\hline parameter & best fit $\pm 1 \sigma$ & $2 \sigma$ range & $3 \sigma$ range \\
\hline$\Delta m_{21}^{2}\left[10^{-5} \mathrm{eV}^{2}\right]$ & $7.55_{-0.16}^{+0.20}$ & $7.20-7.94$ & $7.05-8.14$ \\
$\left|\Delta m_{31}^{2}\right|\left[10^{-3} \mathrm{eV}^{2}\right](\mathrm{NO})$ & $2.50 \pm 0.03$ & $2.44-2.57$ & $2.41-2.60$ \\
$\left|\Delta m_{31}^{2}\right|\left[10^{-3} \mathrm{eV}^{2}\right](\mathrm{IO})$ & $2.42_{-0.04}^{+0.03}$ & $2.34-2.47$ & $2.31-2.51$ \\
$\sin ^{2} \theta_{12} / 10^{-1}$ & $3.20_{-0.16}^{+0.20}$ & $2.89-3.59$ & $2.73-3.79$ \\
$\sin ^{2} \theta_{23} / 10^{-1}(\mathrm{NO})$ & $5.47_{-0.30}^{+0.20}$ & $4.67-5.83$ & $4.45-5.99$ \\
$\sin ^{2} \theta_{23} / 10^{-1}(\mathrm{IO})$ & $5.51_{-0.30}^{+0.18}$ & $4.91-5.84$ & $4.53-5.98$ \\
$\sin ^{2} \theta_{13} / 10^{-2}(\mathrm{NO})$ & $2.160_{-0.069}^{+0.083}$ & $2.03-2.34$ & $1.96-2.41$ \\
$\sin ^{2} \theta_{13} / 10^{-2}(\mathrm{IO})$ & $2.220_{-0.074}^{+0.076}$ & $2.07-2.36$ & $1.99-2.44$ \\
$\delta / \pi(\mathrm{NO})$ & $1.32_{-0.15}^{+0.21}$ & $1.01-1.75$ & $0.87-1.94$ \\
$\delta / \pi(\mathrm{IO})$ & $1.56_{-0.15}^{+0.13}$ & $1.27-1.82$ & $1.12-1.94$ \\
\hline
\end{tabular}

Table 1: Neutrino oscillation parameters summary determined from the global fit [1]. The ranges for inverted ordering refer to the local minimum for this neutrino mass ordering.

\section{The CP-phase}

The main sensitivity to the CP-phase $\delta$ comes from the long-baseline accelerator experiment T2K, although some sensitivity comes also from atmospheric data of Super-Kamiokande and also from the other current accelerator experiment NOvA. These experiments have already some sensitivity on their own, but it increases importantly once combined with the reactor experiments which measure the reactor angle $\theta_{13}$. However, as can be seen in the lower right panel of Fig. 1 a large part of the parameters space remains allowed.

\section{The atmospheric octant}

The mixing angle $\theta_{23}$ is measured by all the long-baseline experiments. The values of the second octant $\left(\sin ^{2} \theta_{23}>0.5\right)$ are degenerate with values in the first octant $\left(\sin ^{2} \theta_{23}<0.5\right)$, which 


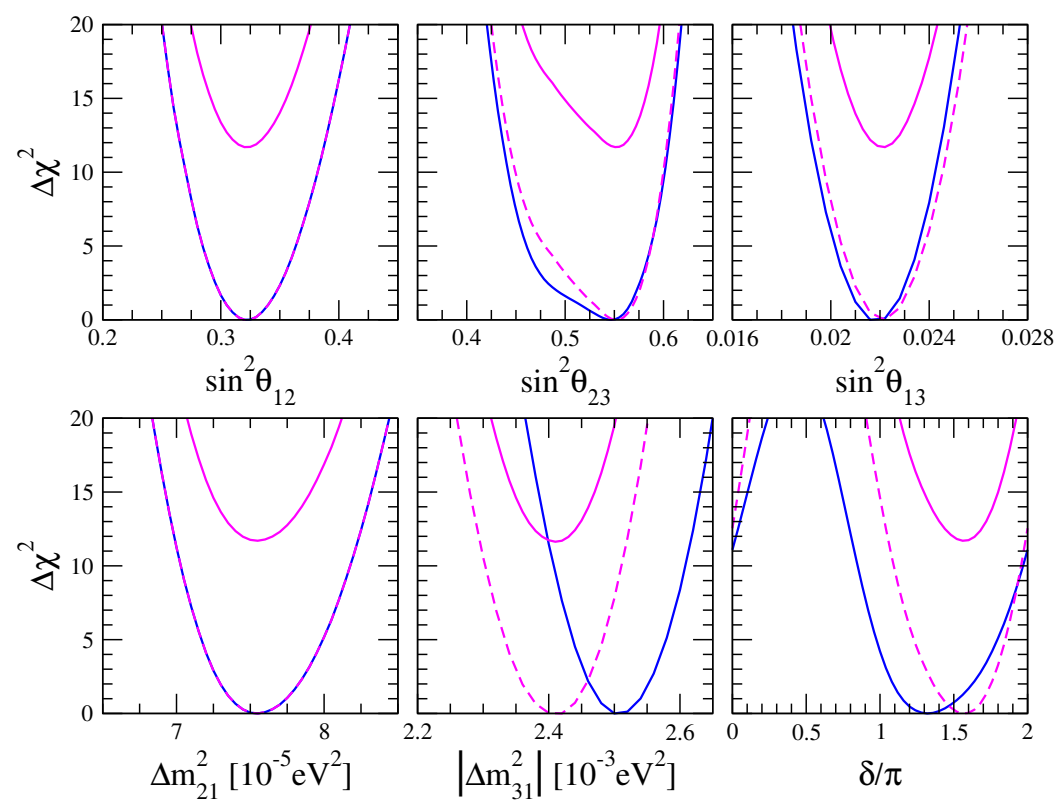

Figure 1: Summary of neutrino oscillation parameters. Blue lines correspond to normal ordering and magenta lines to inverted. The $\Delta \chi^{2}$-profiles for inverted ordering are plotted with respect to the minimum for this neutrino mass ordering (dashed) as well as with respect to the global minimum (solid lines).

makes it very difficult to measure its exact value. The closer the value lies to maximality $\left(\sin ^{2} \theta_{23}=\right.$ 0.5 ), the more difficult it gets to break this degeneracy. While the current best fit lies in the second octant, first octant solutions are still allowed at close to $1 \sigma$ level, as can be seen in the upper middle panel of Fig. 1.

\section{The neutrino mass ordering}

The last unknown is the neutrino mass ordering, referred to as normal if $\Delta m_{31}^{2}>0$ and as inverted if $\Delta m_{31}^{2}<0$. The combination of all oscillation data gives us now a preference for normal ordering of approximately $3 \sigma$, as seen in Fig. 1. To obtain stronger results it is possible to combine these data with observations from decay experiments and Cosmology. However, in doing this one has to be careful in the treatment of priors [2]. Using the most conservative parameterizations we obtain a total preference of about $3.5 \sigma$ in favor of the normal neutrino mass ordering [3].

\section{References}

[1] P. F. de Salas et al., Status of neutrino oscillations 2018: $3 \sigma$ hint for normal mass ordering and improved CP sensitivity, Phys. Lett. B782 (2018) 633 [1708.01186].

[2] S. Gariazzo et al., Neutrino masses and their ordering: Global Data, Priors and Models, JCAP 03 (2018) 011 [1801.04946].

[3] P. F. de Salas et al., Neutrino Mass Ordering from Oscillations and Beyond: 2018 Status and Future Prospects, Front. Astron. Space Sci. 5 (2018) 36 [1806.11051]. 